\title{
VICTORIA PÍRRICA: FODOR Y LA EXPLICACIÓN CIENTÍFICA DE UNA PSICOLOGÍA DE SENTIDO COMÚN
}

\author{
[PYRRHIC VICTORY: FODOR AND THE SCIENTIFIC EXPLANATION OF A PSYCHOLOGY OF \\ COMMON SENSE]
}

\begin{abstract}
Resumen: Contra los molinos del holismo semántico y el neo-empirismo, el Quijote Fodor blande su lanza metafísica del realismo científico. El resultado es una victoria pírrica, puesto que, si bien logra dar una explicación plausible de los mecanismos mentales de fijación de creencias, su reivindicación de una psicología de sentido común debe recurrir al argumento circular de que las propiedades mentales son parte del orden del mundo. Su mérito, no obstante, es indiscutible: no se llega a la verdad científica por la vía de la neurofisiología, sino a través de nuestra cotidiana intencionalidad.
\end{abstract}

Palabras Clave: Creencias; inferencia; mecanismo; mente; realismo científico

\section{INTRODUCCIÓN}

Abstract: Against the windmills of semantic holism and neo-empiricism, Quixote Fodor brandishes his metaphysical lance of scientific realism. The result is a Pyrrhic victory, since, while he manages to provide a plausible explanation for the mental mechanisms of belief fixation, his claim for a common sense psychology must resort to the circular argument that mental properties are part of the order of the world. His merit, however, is indisputable: scientific truth is not reached through neurophysiology, but through our daily intentionality.

KEYwords: Beliefs, inference, mechanism, mind, scientific realism.

$\mathrm{M}$ e propongo realizar una aproximación a la variante fisicalista de la mente que representa Jerry Fodor, coincidiendo con Wakefield (2003) en que su propuesta teórica debe ser vista como un intento de esclarecer, dicho en sentido amplio, la inescrutabilidad en la semántica naturalizada a partir de un esfuerzo estrictamente no analítico. Tal intento queda bastante bien expuesto por Fodor \& Pylyshyn (2014), a propósito del problema de larga data que supone asociar actitudes proposicionales y causación mental: "This is a metaphysical minefield and has been at least since Plato, and it is one in which we don't intend to wander. We will simply take for granted that abstracta are without causal powers; only 'things-in-the-world' (including, in particular, individual states and events) can

* Doctor en Filosofía por la Universidad de Chile. Académico e investigador del área de Informática \& Telecomunicaciones en el Instituto INACAP Chile, sede Temuco, Chile. E-mail: leopoldo.tilleria@inacapmail.cl 
have causes or effects" [Este es un campo minado metafísico y lo ha sido al menos desde Platón, y es uno en el que no tenemos la intención de vagar. Simplemente daremos por sentado que lo abstracto no tiene poderes causales; solo las "cosas en el mundo" (incluidos, en particular, estados y eventos individuales) pueden tener causas o efectos] (p. 6).

Esta sería una primera versión -acertada, desde luego. Otra versión -también correcta- es la que ve en el mentalismo de Fodor una confrontación teórica con las ciencias cognitivas centrada en el problema de la relación entre las representaciones mentales y la semántica composicional, y para la que propone como salida la revitalización de una psicología de sentido común. Pero aún hay otra forma de enfocar el asunto, y es aquella que, en una suerte de justa medieval en la que por un carril se acerca la filosofía blandiendo la lanza del fundamento, y por el otro, y en dirección opuesta, arremete a todo galope -pudiera hasta decirse con una armadura más reluciente- la ciencia y su lanza imbatible de la experiencia, enfrenta a la filosofía y la ciencia en el fangoso terreno de la fijación de creencias.

Pues bien, lo que sugiero es que la relación mente/mundo, es decir, lo que debiera ser el corazón de todo programa de filosofía de la mente, obtiene en el proyecto de Fodor una especificación que tiende a soslayar el tradicional encono epistemológico entre "filósofos" y "científicos", concepto y experiencia. Así, la propuesta de Fodor, a pesar de su escepticismo epistemológico y de su idea de que la adquisición de un concepto se ve mejor como un fenómeno biológico que cognitivo (MARGOLIS \& LAURENCE, 2011), pareciera cumplir con los requisitos indispensables de una teoría filosófica de conceptos que incluya las condiciones que satisfagan una teoría psicológica de conceptos. De manera que, y esto sería imposible en un trabajo introductorio, no abordaré su archiconocida idea de modularidad de la mente o su apasionante teoría del atomismo informacional, sino que discutiré con un poco más de holgura la relación que plantea entre experiencia y contenidos mentales e intentaré demostrar que su idea de una psicología ordinaria contiene, al mismo tiempo, una justificación filosófica y un mecanismo de causación mental que la confirma como psicología de la experiencia.

Probablemente el "ciudadano Fodor" - en la feliz alusión de Cela-Conde y Marty (1991)- haya estado de acuerdo con el título de este trabajo, considerando su asumida condición de l'enfant terrible de la psicología. Se diría que la esencia de su programa fue la crítica, que se especializó en derribar todo tipo de "ortodoxias", especialmente aquellas de cuño neofregeano. Así, por ejemplo, se enfrentó al conductismo de Wittgenstein, Ryle, Skinner y Osgood; desafió las explicaciones experienciales de la adquisición de conceptos de Vygotsky y Piaget; criticó la semántica procedimental de Johnson-Laird y la psicología ecológica de Gibson; desmanteló el holismo del significado y desplegó argumentos quineanos contra la semántica del rol conceptual (REY, 2018, p. 332).

Partiré presentando los principales alcances de la teoría de Fodor en lo relativo a su pretensión justificativa de una psicología de sentido común de creencias/deseos. Enseguida abordaré su fisicalismo no reduccionista, reflejado sobre todo en la creencia de que las propiedades psicológicas, aunque físicamente irreducibles, son propiedades genuinas y tan reales como las propiedades físicobiológicas. Al final discutiré el estatus de no demostratividad de la confirmación científica en el modelo fodoriano y propondré que la isotropía y el quineanismo funcionan como propiedades que vienen a "salvar" a Fodor de una derrota 
estrepitosa en el campo de la inescrutabilidad de las cadenas causales entre los estados mentales.

\section{LA PSICOLOGÍA DE SENTIDO COMÚN COMO EXPLICACIÓN ONTOLÓGICA}

El modelo de mente que postula Fodor en varios de sus textos, pero fundamentalmente en The modularity of mind (1983), no es solo una reacción frente a los avances en las ciencias cognitivas del constructivismo y del asociacionismo computacional, sino en especial una reivindicación de la psicología de sentido común y su entramado intencional de explicaciones (GARCIAA, 2009). El filósofo norteamericano propone una arquitectura de la mente de naturaleza mixta, en parte modular y en parte no modular, pero además con una funcionalidad tripartita: "Concretamente, las inferencias en cuestión tienen como 'premisas' las representaciones transducidas de las configuraciones estimulares proximales, y como 'conclusiones' las representaciones de la naturaleza y la distribución de los objetos distales" (FODOR, 1986, p. 70). Tales inferencias se refieren en realidad a una determinada sintaxis de información que luego, en el nivel del procesamiento central, se transformará en aquel sistema de creencias e hipótesis posibles acerca del estado de cosas en la realidad. De hecho, como luego veremos, este es precisamente el quid de su justificación de una psicología de sentido común, en términos de que Fodor aceptará el carácter inferencial de la percepción, pero bloqueará el argumento que va de allí a la conclusión de que la percepción necesariamente está contaminada por la teoría (ÁVILA, 2000).

En suma, tenemos una mente compuesta descriptiva pero no exhaustivamente por: 1) transductores compilados (sistemas que convierten la estimulación proximal en un determinado formato de información); 2) sistemas de entrada (los únicos sistemas modulares del modelo, y, por lo mismo, encapsulados informacionalmente, encargados de codificar las representaciones mentales que constituyen el ámbito de operación de los mecanismos centrales), y 3) procesadores o sistemas centrales (el "corazón" de la mente y repositorio de lo que el organismo "cree", como dice Fodor, ya sea consciente o subdoxásticamente). En todo caso, lo decisivo del modelo es que para que el razonamiento abductivo sea factible debe ser servido por módulos con bases de datos suficientemente encapsuladas: "In each case, the module in question has access only to a highly restricted and domainspecific database, and it is this sort of informational encapsulation that is supposed to explain the feasibility of abductive inference" [En cada caso, el módulo en cuestión solo tiene acceso a una base de datos muy restringida y específica del dominio, y es este tipo de encapsulación informacional la que se supone que explica la viabilidad de la inferencia abductiva] (RELLIHAN, 2009, p. 319).

Llama la atención que esta mixtura modular/no modular, encapsulamiento/no encapsulamiento, periferia/centro pueda ser sostenida como una arquitectura específica que satisfaga la condición de que en una psicología de las actitudes proposicionales el contenido y los poderes causales se atribuyan a las mismas cosas (FODOR, 1994). Si entiendo bien al estadounidense, la justificación de su modelo se halla no tanto en la estructura modular de la mente, sino en la naturaleza de los procesos mentales que determina, pero más exactamente en la relación creencias/mundo que su teoría defiende. Como enfatiza Dietrich (2001), 
todos los científicos cognitivos, menos uno, sostienen la visión de que la epistemología reside en los conceptos mismos: "But Fodor, lonesome Fodor, the Don Quixote of cognitive science, goes with the latter-concepts lack any epistemic component essentially, but nevertheless participate in epistemic processes in virtue of their semantic nature" [Pero Fodor, el solitario Fodor, el Don Quijote de la ciencia cognitiva, opta por lo último -los conceptos carecen esencialmente de cualquier componente epistémico, pero sin embargo participan en los procesos epistémicos en virtud de su naturaleza semántica] (p. 90).

Contra lo que un importante número de sus adversarios plantea, mi recepción de Fodor considera que su idea de una epistemología naturalizada satisface en buena medida los requerimientos de una prueba de instanciación verdadera respecto de creencias verdaderas acerca del mundo. Su tesis sobre la mente cognitiva tiene la virtud epistemológica de no excluir, por muy metafísico que sea el fundamento de su realismo intencional, los componentes prácticos (o empíricos, si se quiere) en las explicaciones del problema de la composicionalidad de la mente, no obstante, su reformulación ontológica de la relación entre observación y experiencia. Dicho en sus propias palabras: "Toda psicología que sea realista con relación a lo mental reconoce, ipso facto, los poderes causales de lo mental" (FODOR, 1994, p. 31). Como se advierte, su argumentación se centra en el postulado del fisicalismo no reduccionista como explanans de los procesos cognitivos. En efecto, para Fodor se trata de hacer consistente su tesis filosófica madre de que una psicología explícita con poderes predictivos debe poder explicar ontológica y no epistemológicamente la relación entre los hechos y nuestros sistemas de creencias y actitudes, a partir de la configuración de nuestros estados mentales como dispositivos de causación mental.

De esta laya, su Teoría Representacional de la Mente se muestra "capaz de explotar el paralelismo existente entre las relaciones causales entre los eventos mentales y las relaciones semánticas que valen entre sus objetos proposicionales" (BRUNSTEINS, 2003, p. 40). En palabras sencillas: creemos ciertas cosas sobre el mundo o deseamos otras de él, o bien tenemos determinadas intenciones que causan nuestra conducta en el entorno respetando sensatamente la razonabilidad de este conglomerado de deseos y creencias. Conviene aclarar el compromiso de Fodor con el programa fisicalista para determinar en qué medida la experiencia juega o no un papel relevante en la psicología de sentido común que pretende reivindicar.

\section{FisicAlismo no REDUCCIONISTA}

Antes de establecer conclusiones más de fondo acerca de nuestra suposición inicial, que sugiere que la teoría de Fodor satisface condiciones de plausibilidad empírica en su perspectiva de explicación científica de la psicología de sentido común, creo necesario describir esquemáticamente su fisicalismo no reduccionista. Como se sabe -y probablemente esta sea la "eterna" espada de Damocles que penderá sobre su cabeza-, la teoría de la mente de Fodor adhiere radicalmente al dualismo cartesiano. Mente y cuerpo corren por carriles distintos, negando de plano toda posibilidad de monismo, conexionismo u holismo cognitivo. Este dualismo se "refleja en la creencia de que las propiedades psicológicas, aunque físicamente 
irreducibles, son propiedades genuinas, tan reales como las propiedades físicobiológicas" (KIM, 2014, p. 239). Siguiendo la tesis de la realizabilidad múltiple de Putnam (1967), Fodor mantiene el supuesto de que los estados mentales pueden materializarse en sistemas y entidades físicos, sin riesgo de dar explicaciones físicas, puesto que dicha materialización se realizaría sobre base del principio de isomorfismo funcional (MEDINA, 2010).

Esta instanciación, en todo caso, no debe vulnerar el principio metafísico según el cual solo las relaciones con entidades reales y efectivas afectan a los poderes causales de algo (HERMIDA, 1993), lo cual se verifica mediante ciertas condiciones no negociables de dicha teoría: que analice la noción de contenido en términos de las de "propiedad", "causa", "ley natural", etc., y que haga esto sin recurrir a expresiones intencionales o semánticas (ACERO, 2006). En consecuencia, lo característico del fisicalismo es su afirmación de que las propiedades mentales son propiedades físicas, o al menos su suposición de que están constituidas y son realizadas por propiedades físicas o son supervenientes de ellas (CASTILLO, 2018), independiente de que su explicación pueda ser proporcionada por ciencias especiales distintas de la física. Fodor defenderá un fisicalismo múltiple, en el que las propiedades mentales son instanciables en una multiplicidad de estructuras físicas disímiles y, por lo mismo, solo una disyunción abierta, extensa y artificial de predicados físicos que denoten todas las posibles realizaciones físicas de una propiedad mental puede constituir el predicado físico reductor de la clase mental (MORALES, 2013).

Es decir: la mente sería una entidad propia que determina ciertos comportamientos o expresiones lingüísticas y no otras, en razón justamente de las propiedades causales que caracterizan los procesos mentales y, por ende, el modo de fijación de ciertas creencias y actitudes proposicionales.

Ahora bien, el que la mente sea una entidad real implica el principio fisicalista de que es imposible establecer correlaciones entre tipos de estados mentales y tipos de estados físicos, y, por tanto, no hay posibilidad de formular leyes puente que permitan reducir la psicología a otras disciplinas científicas más básicas (PEDACE, 2001). Fodor rompe con aquellas tesis materialistas que tendían a identificar estados mentales con estados cerebrales, al estilo de que cada tipo de estado mental se correspondería con un determinado tipo de estado cerebral. Tal objeción, se expresa específicamente en el argumento que sostiene que, aunque aceptáramos que una creencia determinada se identifica con un cierto estado del cerebro, parece demasiado restrictivo suponer que todo individuo que tuviera la misma creencia debiera tener exactamente el mismo tipo de evento neurológico (AGUINALDE, 2018). De modo que para no quedar atrapado en el argumento materialista de la identidad tipo de estado mental/tipo de estado cerebral, Fodor propone cambiar la naturaleza de la instanciación mental (podría decirse, el "evento" metafísico) y cambiar el componente tipo por el de caso, con el argumento, como indica Aguinalde (2018), de que lo único posible de afirmar en términos fisicalistas es que cada caso o instancia particular de un estado mental es idéntico a algún caso de algún tipo de estado del cerebro, cualquiera sea ese tipo.

Dicha teoría (token-token identity theory) quedaría formulada así: "Los procesos mentales son secuencias causales de muestras/instanciaciones (tokens) de representaciones mentales en virtud de su forma (sintaxis) y preservando su contenido (semántica)" (RODRÍGUEZ, 2006, p. 20). Lo que hay detrás de esta 
versión de la mente cognitiva es la convicción de que la psicología debe quedar reducida a la condición de ciencia especial, irreductible a un programa sistémico ya sea en sus variantes ciencias cognitivas o neurociencias. Y esto, puesto que el error de los programas materialistas y conexionistas, piensa Fodor, ha consistido en creer que el problema de la naturaleza de la cognición radica en presupuestos epistemológicos, cuando en realidad el problema de la verificabilidad de los estados mentales es esencialmente metafísico. Incluso si los eventos mentales son eventos neurológicos, de esto no se sigue que los predicados de clase natural de la psicología sean coextensivos con los predicados de clase natural de cualquier otra disciplina, incluida la física (PEDACE, 2001).

\section{LA NO DEMOSTRATIVIDAD DE LA CONFIRMACIÓN CIENTÍFICA}

Hasta aquí se ha pergeñado la teoría de Fodor como una especie de caja negra en cuanto a la arquitectura causal/representacional de los estados mentales. Tal cosa significa que la justificación de su teoría de la mente parte, primero, del supuesto de que la heurística de los sistemas centrales, en cuanto a la elaboración de las hipótesis más probables acerca del estado de cosas en el mundo, depende mayormente de procesos inconscientes de los que se sabe muy poco cómo funcionan; y segundo, de que su teoría debe aceptar la inobservabilidad de buena parte de sus relaciones causales. En el caso de la psicología de sentido común, esto se traduciría en la inescrutabilidad de las cadenas causales que conectan unos estados mentales con otros (HERMIDA, 1993). Lo que arguye Fodor a continuación es la tesis de que la fijación de creencias científicas por medios no demostrativos (la función primordial de los sistemas centrales) está determinada por dos propiedades de confirmación: la isotropía y el quineanismo.

Ambas propiedades, qua procesos de inferencia racional no demostrativa, lo que hacen es fungir como mecanismos de analogía mediante los cuales Fodor compara los propios mecanismos de fijación de creencias con los mecanismos de confirmación de las teorías científicas: "Para inferirse algo sobre el funcionamiento de los procesos mentales, a partir de los procesos explícitos de inferencia no demostrativa nos hemos de basar en lo que sabemos acerca de la inferencia empírica en la ciencia" (PADILLA-GÁLVEZ, 1992, p. 141). Como ahora espero demostrar, esta "jugada" justificativa de Fodor parece tener un alcance decisivo en nuestra conjetura que conecta sus enunciados filosóficos sobre la mente con la idea de explicación a nivel empírico de la psicología de sentido común.

La isotropía funciona como un principio normativo por medio del cual los datos relevantes para confirmar una hipótesis científica pueden provenir de cualquier área del universo de verdades empíricas o demostrativas previamente establecidas. Es un principio que le permite a la ciencia ir avanzando en su tarea de construcción teórica en la medida en que los nuevos datos confirmatorios, provenientes de disciplinas arbitrarias, van desechando hipótesis no confirmadas y validando los enunciados que se adecuan a las nuevas verdades empíricas. Ahora, su "descubrimiento" le permite a Fodor sortear una de las mayores dificultades de su teoría de la mente: la inexistencia de pruebas directas que expliquen el funcionamiento no encapsulado de los sistemas centrales en su faena de fijación de creencias. En efecto: "La isotropía de la confirmación es una de las propiedades de 
la inferencia no demostrativa, porque el objetivo de este tipo de inferencia es determinar el grado de verdad de un mecanismo causal -el mundo- cuyo funcionamiento ignoramos de manera arbitraria" (FODOR, 1986, p. 149). Isotrópicamente, los sistemas centrales (no encapsulados, no modulares y no computacionales) aspiran a la globalidad cognitiva (ESCALONILLA, 2015) bajo la hipótesis de solución provisoria del conocido "problema del marco". Este consiste, grosso modo, en la tesis filosófica sobre el problema del mecanismo por medio del cual los procesos cognitivos determinan qué información de entre todo el repertorio disponible es relevante para una tarea determinada.

Fodor cree que las operaciones cognitivas de los sistemas centrales requieren de una búsqueda exhaustiva entre toda la información que posee el sistema, lo cual es extremadamente implausible (SILENZI, 2015). Tal implausibilidad es resuelta precisamente por el principio de isotropía, el que vendría "como caído del cielo" a resolver el problema epistemológico de la inconmensurabilidad entre teorías científicas pertenecientes a paradigmas distintos. En tal sentido, la isotropía solo aportaría pruebas de plausibilidad global, aunque, como reconoce Chow (2016), Fodor nunca pretendió hacer más que esto: "And since scientific minds aren't unlike the minds of ordinary folk, ordinary cognition is plausibly global in this sense. Of course, none of this constitutes demonstrative proof that quotidian cognition is global -it remains a plausibility argument" [Y dado que las mentes científicas no son diferentes a las mentes de la gente común, la cognición ordinaria es plausiblemente global en este sentido. Por supuesto, nada de esto constituye una prueba demostrativa de que la cognición cotidiana sea global -sigue siendo un argumento de plausibilidad] (p. 175).

A su vez, el quineanismo establece que el grado de confirmación que se atribuye a una determinada hipótesis es sensible a las propiedades del sistema de creencias en su totalidad (FODOR, 1986). Desde luego, la clave aquí es la fórmula "determinada hipótesis", en el sentido de que a diferencia de la isotropía que presenta un alcance global, el carácter quineano está referido a confirmaciones locales (determinadas) de la teoría científica en cuestión (el sistema de creencias). El argumento es el siguiente: cada hipótesis sobre fenómenos no observables debe implicar ciertas predicciones sobre fenómenos observables, de donde tales implicaciones tienen que ser válidas en virtud de la semántica de los términos teóricos contenidos en tales hipótesis (FODOR, 1986). En suma: la isotropía y el quineanismo son propiedades que justifican filosóficamente la conexión entre los procesos centrales de fijación de creencias y los procesos de inferencia racional no demostrativa, o, si se quiere, entre casos empíricos e hipótesis científicas disponibles en nuestro corpus de datos mentales.

Todo indica que el uso de estos criterios de justificación -más allá de que respondan disciplinadamente a su famosa «Primera ley de Fodor de la inexistencia de la ciencia cognitiva»-, representan para Fodor una victoria pírrica no solo respecto del consabido problema del marco, sino especialmente en relación al problema metafísico que supone en la construcción de teorías el que no haya modo de delimitar los tipos de recursos informativos que puedan afectar tales procesos cognitivos centrales. Su tesis de fijación de creencias científicas apela subdoxásticamente al sentido común y endosa a la psicología ordinaria la tarea de proveer explicaciones científicas plausibles mediante la atribución de poderes causales a nuestros estados intencionales. La confirmación de las creencias 
científicas, por tanto, no puede sino ser a posteriori, justamente a partir de la provisión de determinadas clases de datos que susciten las combinaciones de los enunciados que mejor se adecúen a nuestro arsenal de información disponible. Así: Los datos de la experiencia determinarán todo el movimiento del sistema de fijación de creencias a partir del conjunto de verdades empíricas, formando, nolens volens, la base de los procesos explícitos de inferencia no demostrativa.

Es lo mismo que razona Antony (2020) sobre la relación experiencia/contenido mental: "Learning is a causal, computational process relating experience to a novel mental state such that, first, there is a rational relation between the experience and the content of the subsequent mental state, and second, the state-transitions between the experience and the end mental state mirror rational relations" [El aprendizaje es un proceso causal, computacional, que relaciona la experiencia con un estado mental novedoso tal que, primero, existe una relación racional entre la experiencia y el contenido del estado mental subsiguiente, y segundo, las transiciones de estado entre la experiencia y el estado mental final reflejan las relaciones racionales] (p. 46). Sin embargo, si dejáramos las cosas así Fodor quedaría convertido en un perfecto neo-empirista, algo que definitivamente no es. A pesar de su radicalismo epistemológico, prima en su planteamiento el intento de fijar un justo medio respecto de la distinción observación/inferencia y entre la neutralidad pretendida por los positivistas y la imposibilidad de acceder a los hechos postulada por los constructivistas (AGUIRRE Y JARAMILLO, 2013). Como lapidariamente afirma el filósofo estadounidense, lo que exige el argumento justificatorio no es que algunas de las creencias sean incuestionables sino, como máximo, que algunas de ellas no sean cuestionadas de facto.

\section{Conclusiones}

La filosofía de la mente de Fodor funciona también como filosofía de la ciencia solo si la entendemos como un intento de explicación científica de una psicología de las actitudes proposicionales. Vale decir, únicamente si esta psicología de sentido común es vista al mismo tiempo como psicología de la experiencia. Apelando a su materialismo mental, la psicología empírica defendida por Fodor no solo tiene un alto grado de probabilidad de ser verdadera, sino que refleja la idea de una perfecta Navaja de Ockham respecto de la pregunta "ancestral" de las ciencias cognitivas por la conexión entre los hechos y nuestros sistemas de creencias y actitudes. Inopinadamente, la isotropía y el quineanismo, como criterios de justificación ad hoc, expresan la victoria pírrica de Fodor respecto de la imposibilidad metafísica de delimitar los tipos de recursos informativos que puedan afectar nuestros mecanismos de fijación de creencias empíricas. El problema podría resolverse si apelamos al sentido común no solo como un explanans, sino que, yendo incluso más allá de Fodor, al modo como lo ha querido ver el mismo McCarthy (1996), es decir, si el programa que lo posee es capaz de deducir automáticamente y por sí mismo una clase suficientemente amplia de consecuencias no neurológicas inmediatas de cualquier cosa que se le diga y de lo que ya sabe. Tal programa parece ser la mente.

\section{REFERENCIAS}


ACERO, J. J. (2006). La Naturalización de La Intencionalidad. Episteme NS, 26(1), 1-40. Disponible en: <https://tinyurl.com/yxvp6vv8>. Acceso en: 26 de diciembre de 2020.

AGUINALDE, I. (2018). Problemas del materialismo en la filosofía de la mente. Seminario "Persona, mente y cerebro". Instituto de Filosofía, Universidad Austral.

AGUIRRE, J. y JARAMILLO, L. (2013). Tesis de la carga teórica de la observación y constructivismo. Cinta Moebio, 47, 74-82. Disponible en: $<$ https://tinyurl.com/y32d3cv4>. Acceso en: 20 de noviembre de 2020.

ANTONY, L. (2020). Not rational, but not brutely causal either: A response to Fodor on concept acquisition. Theoria, 35(1), 45-57. Doi: https://doi.org/10.1387/theoria.21031

ÁVILA, I. (2000). La modularidad de la mente y el relativismo epistemológico. Ideas y Valores, 49(112), 37-65. Disponible en: <https://tinyurl.com/y4v9zw24>. Acceso en: 02 de noviembre de 2020.

BRUNSTEINS, P. (2003). La psicología del sentido común después del materialismo eliminativista. Epistemología e Historia de la Ciencia, 9(9), 40-45. Disponible en: $<$ https://tinyurl.com/y3cvfjz2>. Acceso en: 25 de octubre de 2020.

CASTILlO, S. (2018). Causalidad y emergencia. Crítica, 50(150), 27-41. Doi: 10.22201/iifs. 18704905 e.2018.14

CELA CONDE, C. y MARTY, G. (1991). El ciudadano Fodor no existe: Estructura y función en los procesos cognitivos. Psicothema, 3(1), 231-241. Disponible en: $<$ https://tinyurl.com/y2w3bfod $>$. Acceso en 04 de enero de 2021.

CHOW, S. (2016). Fodor on global cognition and scientific inference. Philosophical Psychology, 29(2), 157-178. Doi: http://dx.doi.org/10.1080/09515089.2015.1013208

DIETRICH, E. (2001). Concepts: Fodor's little semantic BBs of thought. A critical look at Fodor's theory of concepts. Journal of Experimental \& Theoretical Artificial Intelligence, 13(2), 89-94. Doi: 10.1080/09528130117948

ESCALONILLA, A. (2015). El origen del lenguaje desde la psicobiología contemporánea: Salto lingüístico o gradualismo [Tesis doctoral]. Universidad Complutense de Madrid.

FODOR, J. (1986). La modularidad de la mente. Madrid: Morata.

FODOR, J. (1994). Psicosemántica. Madrid: Tecnos.

FODOR, J. \& PYLYSHYN, Z. (2014). Minds Without Meanings : An Essay on the Content of Concepts. Cambridge, Massachusetts: The MIT Press.

GARCÍA, O. (2009). Reflexiones en torno a la filosofía de la mente. Cultura, 23(23), 101-120. Disponible en: <https://tinyurl.com/y26yu9lv>. Acceso en: 10 de enero de 2021.

HERMIDA, P. (1993). La Psicosemántica de Jerry A. Fodor. Endoxa, Series Filosóficas (1), 359372. Disponible en: <https://tinyurl.com/ycm8ft9h>. Acceso en: 14 de octubre de 2020.

KIM, J. (2014). El fisicalismo no reduccionista y su problema con la causalidad mental. Ideas y $\begin{array}{llll}\text { Valores, } & \text { LXIII } & \text { 235-259. } & \text { Doi: }\end{array}$ http://dx.doi.org/10.15446/ideasyvalores.v63n155.44762

MARGOLIS, E. \& LAURENCE S. (2011). Learning Matters: The Role of Learning in Concept Acquisition. Mind \& Language, 26(5), 507-539. Doi: 10.1111/j.1468-0017.2011.01429.x

McCARTHY, J. (1996). Concepts of Logical AI. Computer Science Department. Stanford University. Disponible en: <https://tinyurl.com/63gw3>. Acceso en: 20 de octubre de 2020.

MEDINA, N. (2010). Realidad mental en la ciencia cognitiva. Revista de Investigación en Psicología, 13(1), 209-220. Disponible en: <https://tinyurl.com/y3h6rtlh>. Acceso en: 12 de noviembre de 2020.

MORALES, J. (2013). Fodor y Kim en torno a la posibilidad de las ciencias especiales, la realizabilidad múltiple y el reduccionismo. Revista Colombiana de Filosofía de la Ciencia, 13(27), 63-84. Disponible en: <https://tinyurl.com/y2jxdvx3>. Acceso en: 16 de enero de 2021.

PADILLA-GÁLVEZ, J. (1992). Confirmación isotrópica-quineana reconsiderada. Taula, 15, 137146. Disponible en: <https://tinyurl.com/y474fsxa $>$. Acceso en: 14 de octubre de 2020.

PEDACE, K. (2001). De la implausibilidad de los fisicalismos no reduccionistas. El monismo anómalo de D. Davidson y la teoría representacional de la mente de J. Fodor [Tesis de grado]. Universidad de Buenos Aires. 
RELliHAN, M. (2009). Fodor's riddle of abduction. Philosophical Studies, 144(2), 313-338. Doi: $10.1007 / \mathrm{s} 11098-008-9212-6$

REY, G. (2018). Remembering Jerry Fodor and his work. Mind \& Language, 33, 321-341. Doi: 10.1111/mila.12206

32 Universidad de Chile.

RODRÍGUEZ, M. (2006). Sobre conceptos. Atomismo Informacional. [Tesis de Magister].

SILENZI, M. I. (2015). ¿En qué consiste el problema de marco? Confluencias entre distintas interpretaciones. Eidos (22), 49-80. Doi: http://dx.doi.org/10.14482/eidos.22.6381

WAKEFIELD, J. (2003). Fodor on Inscrutability. Mind \& Language, 18(5), 524-537. Doi: $10.1111 / 1468-0017.00241$ 